\title{
Rose-Marie Arbour
}

PhD, histoire de l'art, professeure associée, Département d'histoire de l'art, UQÀM

(1987)

\section{“Quelques hypothèses pour une histoire de l'art des femmes, 1965-1985”}

Un document produit en version numérique par Jean-Marie Tremblay, bénévole, professeur de sociologie au Cégep de Chicoutimi Courriel: jean-marie tremblay@uqac.ca

Site web pédagogique : http://www.uqac.ca/jmt-sociologue/

Dans le cadre de la collection: "Les classiques des sciences sociales"

Site web: http://classiques.uqac.ca/

Une collection développée en collaboration avec la Bibliothèque Paul-Émile-Boulet de l'Université du Québec à Chicoutimi Site web: http://bibliotheque.uqac.ca/ 
Cette édition électronique a été réalisée par Jean-Marie Tremblay, bénévole, professeur de sociologie au Cégep de Chicoutimi à partir de :

\section{Rose-Marie Arbour}

[PhD, histoire de l'art, professeure associée, Département d'histoire de l'art, UQÀM.]

\section{"Quelques hypothèses pour une histoire de l'art des fem-} mes, 1965-1985”.

Un article publié dans Le monde selon Graff, 1966-1986, Ed Graff, Montréal, 1987, pp. 575-589.

[Autorisation accordée le 31 décembre 2006 par Mme Arbour de diffuser toutes ses publications dans Les Classiques des sciences sociales.]

Courriel : arbour.rose-marie@uqam.ca

Polices de caractères utilisée :

Pour le texte: Times New Roman, 14 points.

Pour les citations : Times New Roman, 12 points.

Pour les notes de bas de page : Times New Roman, 12 points.

Édition électronique réalisée avec le traitement de textes Microsoft Word 2004 pour Macintosh.

Mise en page sur papier format : LETTRE (US letter), 8.5’’ x 11’’)

Édition numérique réalisée le 11 mai 2007 à Chicoutimi, Ville de Saguenay, province de Québec, Canada. 


\section{Table des matières}

Introduction

Le post-automatisme: situation et rôle des femmes

Les aspirations et manifestations d'un Québec «underground».

Les femmes-artistes se regroupent

Les années 70: cinq propositions

Constance de la démarche figurative chez les femmes-artistes depuis vingt-cinq ans

Le geste dans l'abstraction

Sculpture, environnement, installation

Photo, vidéo, performance 
Rose-Marie Arbour

“Quelques hypothèses pour une histoire de l'art des femmes, 1965-1985”.

Un article publié dans Le monde selon Graff, 1966-1986, Ed Graff, Montréal, 1987, pp. 575-589.

\section{Introduction}

$\underline{\text { Retour à la table des matières }}$

Au milieu des années 60, Guy Viau définissait la double mission de l'artiste québécois: celle d'être une synthèse entre le NouveauMonde et l'Ancien, celle d'être un modèle singulier dont le courage, le réalisme, la capacité de vision peuvent seuls permettre de réaliser cette synthèse de l'Europe et de l'Amérique: «... et paradoxe, l'illustration de ce courage de cette virilité nous vient en particulier des femmes-peintres... ${ }^{1}$ » Selon cet auteur, au Canada même, ce sont «les peintres du Québec qui donnent le ton». On n'aurait pu indiquer plus vigoureusement le sens du développement de l'art au Québec et sa place dans l'ensemble des courants artistiques au Canada.

Vingt ans plus tard, une telle affirmation provoque un sourire contraint car ni les artistes québécois ni les femmes-artistes n’ont réalisé cette mission historique à laquelle Guy Viau les avait conviés. Yves Robillard a fait une analyse des causes de ce rendez-vous raté des artistes québécois au sein de la scène artistique canadienne. Quant

1 Guy Viau, La peinture moderne au Canada-français, Ministère des Affaires culturelles, 1964, p. 85. 
aux femmes-artistes, aucune recherche approfondie n'est encore venue éclairer leur rôle et leur position en tant qu'artistes dans le champ de l’art québécois actuel.

Afin d'évaluer rapidement l'apport des femmes-artistes au cours de cette période, je propose une lecture qui, je l'espère contribuera à faire entendre certains sons, à saisir certaines portées, à conjuguer certaines voix qui ne sont pas nécessairement évidentes au premier abord. À ce titre, le projet de parler séparément des femmes n’en est pas un plus critiquable qu'un autre; il est même toujours nécessaire car la grande utopie visant à abolir les inégalités envers les femmes ne s'est pas réalisée même si depuis une dizaine d'années elle a paru s'approcher d'une certaine concrétisation. Plutôt que d'inégalités à dissoudre, il faut davantage viser les hiérarchies qui sont à l'origine d'exclusions et d'oppressions de toutes sortes. En effet, la situation générale des femmes dans les domaines décisionnels et dans ceux de la création en général se résume encore à maintenir la tête hors de l'eau si bien qu'il y ait, ça et là, quelques nageuses d'exception.

Problématiser l'histoire de l'art des femmes pourrait consister entre autres à situer systématiquement leur œuvres par rapport à celles des autres artistes (hommes) reliés à tel mouvement ou à tel courant et à en saisir la particularité tant sur le plan esthétique que dans les domaines de l'imaginaire auxquels elles réfèrent. La recherche n'est pas encore faite mais je me permettrai d'émettre ici quelques hypothèses, d'avancer certains faits qui, s’ils sont sujets à critiques, n'en permettent pas moins d'aborder par un biais autre que strictement chronologique, l'art des femmes. Réintégrer les femmes-artistes dans l'histoire ne relève en effet pas seulement du redressement d'un ordre chronologique par un autre. Cela consiste surtout à comprendre pourquoi ces artistes avaient été minimisées ou ignorées et ce que ces omissions révèlent de la nature d'un mouvement ou de la valeur du discours des historiens et critiques qui ont participé ou contribué, consciemment ou pas, à normaliser ces oublis. 
Nous aborderons donc cette «histoire» sous des angles divers: soit par le biais de l'importance du geste et de la gestualité chez les femmes-artistes, soit par l'intérêt marqué pour un art à «contenu» où l'expérience personnelle et l'autobiographie sont systématiquement incluses, la tendance à la communication sans pour autant faire de l'œuvre d'art un simple relais. En général, les femmes-artistes sont délaissé la voie formaliste réfractaire à tout contenu et la figuration narrative a été privilégiée chez plusieurs d'entre elles depuis les années 60.

Je tenterai de cerner les raisons de l'absence des femmes de certains mouvements et courants en art mais je ne prétends pas déterminer la position réelle des femmes-artistes dans le champ de l'art québécois actuel. Ce texte est un bref tour d'horizon dont l'objectif est davantage d'ouvrir des perspectives que de bloquer des voies.

\section{Le post-automatisme: situation et rôle des femmes}

$\underline{\text { Retour à la table des matières }}$

L'art abstrait des Plasticiens souleva toutes sortes d'oppositions de la part des jeunes artistes — dont des femmes — au cours des années 60. Ces dernières n'adhérèrent pas à ce mouvement, à l'exception de Rita Letendre. Même si chronologiquement il avait eu son origine au Québec dès les années 50, le groupe des Plasticiens s'était ultérieurement retrouvé sous influence américaine suite à une participation québécoise (Molinari,...) à l'exposition «The Responsive Eye» en 1965 au Museum of Modern Art de New York.

Par ailleurs, au cours des années 60, on sait l'opposition de certains milieux artistiques américains (dont celui des femmes-artistes) à la 
volonté hégémonique du courant formaliste, particulièrement celui chapeauté par le critique d'art Clément Greenberg. Lucy Lippard affirmait ainsi que l'apport le plus important des femmes à l'art du futur aura été son absence de contribution au formalisme ${ }^{2}$. En effet aucune femme n'adhéra au mot d'ordre: «What you see is what you see» formulé par l'artiste américain Frank Stella qui consacrait la stricte autoréférentialité de l'art et l'exclusion systématique de tout ce qui n'était pas spécifique à la matérialité de la peinture.

Au Québec, entre 1955 et 1965, une forme d'abstraction lyrique avait été largement pratiquée par les femmes-artistes, l'émotion, la gestualité et l'expressivité de la couleur formant un tout. Marcelle Ferron, l'une des signataires du Refus Global en 1948, fut une précurseure. Cette artiste eut une présence continue sur la scène artistique: la force de son geste, l'énergie qui s'en dégageait et qui a déterminé l’organisation formelle de ses toiles, répondait à l'idée qui était à la base du mouvement automatiste. À la fin des années 60, elle s'engagea dans un travail de réconciliation de l'art et de la technologie en participant à la formation du Groupe Création où était sollicité le travail conjoint de l'artiste et de l'architecte, de l'artiste et de l'ingénieur, pour une meilleure intégration de l'art à l'environnement.

Les nombreuses verrières réalisées par Marcelle Ferron au Québec et au Canada depuis le milieu des années 60 ont témoigné de cette volonté de mettre l'art sur la place publique et de s'engager dans l'exploration des rapports entre art et technologie.

Une autre femme s'est manifestée quasiment sans interruption sur la scène artistique depuis les années 50: Françoise Sullivan, autre signataire du Refus Global, eut une pratique artistique polyvalente.

2 Lucy Lippard, «Sweeping Exchanges: The Contribution of Feminism to the art of the 1970's», Art Journal, automne-hiver 1980. L'auteure écrit: «the 1970's might not have been 'pluralist' at all if women artists had not emerged during that decade to introduce the multicolored treads of female experience into the mal fabric of modern art». 
Danseuse, sculpteure, peintre, elle a participé à plusieurs courants d'art actuel, utilisant divers médiums selon l'exigence de ses options plastiques et disciplinaires. Elle se situe au sein de courants d'avantgarde auxquels la polyvalence de sa pratique l'ont rendue particulièrement apte à aborder les diverses problématiques et méthodes (art conceptuel, performance,...). La notion de rituel a pris un sens particulier chez elle, il a sous-tendu son travail en sculpture: la croissance, la notion d'éternel retour, les mythes antiques, sont des thèmes qui ont fondé l'ensemble de son travail artistique et créateur que ce soit en danse, en sculpture, en performance, en peinture ${ }^{3}$.

Dans les années 60, il faut rappeler les pôles d'attraction extérieurs au Québec (Paris et New York) qui contribuèrent à dichotomiser les tendances stylistiques et idéologiques dans les arts visuels. À New York, les Plasticiens avaient reçu une certaine reconnaissance qui fut de très courte durée. À Paris, étaient exposés les artistes québécois qui y vivaient déjà: depuis les années 50 , ce fut le cas entre autres pour Marcelle Ferron. En 1961, une exposition «La Nouvelle École de Montréal ${ }^{4} \gg$ qui avait réuni cinq artistes dont deux femmes, constitua une tentative marquée d'exportation de l'art québécois outre-mer au sein de laquelle les femmes étaient relativement bien représentées.

Au début des années 60, à la Galerie nationale du Canada, à Ottawa, avait eu lieu une exposition regroupant des membres de l'Association des peintres non figuratifs de Montréal qui incluait huit femmes sur un total de vingt-deux exposants, et en 1965, à quelques exceptions près, les mêmes artistes faisaient l'objet d'une exposition

3 En 1981, sa rétrospective au Musée d'art contemporain de Montréal confirmait la place unique qu'elle a tenue et tient toujours au sein du champ artistique québécois.

4 Exposition organisée par Jean Cathelin à la Galerie Namber. Elle incluait deux femmes: Tobie Steinhouse et Marcelle Maltais, aux côtés de Réal Arsenault, Germain Perron, Richard Lacroix. 
sous le titre «La femme imagiste» (Galerie de l’Étable, Musée des beaux-arts de Montréal) ${ }^{5}$.

C'est dans ce contexte somme toute positif pour les femmespeintres que l'affirmation de Guy Viau, face au rôle de pointe joué par les femmes dans le champ artistique québécois, s’explique.

On peut affirmer que les femmes-peintres se situèrent hors l'orbite des Plasticiens dont la reconnaissance officielle fut assurée dès le milieu des années 60; d'autres se tourneront vers des problématiques liées à la synthèse des arts (Micheline Beauchemin, Marcelle Ferron, Françoise Sullivan, Jeanne Renaud) à une période où l'intérêt grandissant pour les rapports entre l'art et l'environnement favorisait l'émergence des groupes interdisciplinaires et des intérêts polyvalents.

L'absence de femmes du groupe des Plasticiens (à l'exception de Rita Letendre) s'explique dans un tel contexte d'éclatement des disciplines et des hiérarchies entre les différentes pratiques qui particularisa le travail artistique de nombreuses femmes. Ce fut également le cas d'un nombre important de jeunes artistes dont l'objectif était le rapport de l'art au public et à l'environnement, l'expression au sein de leur art de leur réalité à la fois personnelle et collective; cela entraîna l'implication sociale de plusieurs d'entre eux à la fin des années 60 et au début des années 70 .

5 Au début des années 60 à la Galerie nationale du Canada (Ottawa) avait eu lieu une exposition regroupant vingt-deux membres de l'Association des peintres non figuratifs de Montréal dont huit femmes: Henriette Fauteux-Massé, Marcelle Ferron, Rita Letendre, Laure Major, Kittie Bruneau, Suzanne Meloche, Suzanne Rivard, Tobie Steinhouse. À l'exposition de Montréal (Galerie de l'Étable du Musée des beaux-arts de Montréal, 1965) s’ajoutèrent Françoise Sullivan et Monique Voyer aux peintres mentionnées plus haut. 


\section{Les aspirations et manifestations d'un Québec «underground».}

Retour à la table des matières

La période artistique répertoriée par la publication en trois volumes intitulée Québec underground ${ }^{6}$ est allée de 1962 à 1972; elle fut réalisée sous l'angle d'une conception de l'art conçue comme activité ludique et de participation où la préoccupation pour une intégration de l’art à la société était primordiale.

Curieusement, cette volumineuse publication n'a pas retenu l'activité des femmes qui commencaient alors à se manifester dans une optique d'implication politique de leur pratique artistique. Ainsi est à peine mentionnée l'activité polyvalente d'Irène Chiasson qui dès 1964 travaillait dans des spectacles audio-visuels, à réunir la poésie, la danse et le théâtre, à créer un environnement global. Ses gonflables, en ce sens, réalisaient une conception englobante, éphémère, d'un environnement total et facilement déplaçable. Dans une perspective plus politisée, le groupe Mauve ${ }^{7}$ avait réalisé une vitrine au grand magasin Dupuis Frères (rue Sainte-Catherine à Montréal) où était dénoncée l'image de la femme objet et incitation à la consommation, dans le cadre d'une exposition du groupe «Montréal — ou —», présentée au Musée des beaux-arts de Montréal à l'été 1972. On constate que les groupes «underground» ont inclus très peu de femmes dans leur entreprise de subversion.

6 Quebec Underground, 1962-1972, Les Éditions Médiart, Montréal, 1973. 3 volumes (épuisés).

7 Le Groupe Mauve était composé de Catherine Boisvert, Ghislaine Boyer, Céline, Claudette et Thérèse Isabel, Lise Landry, Lucie Ménard. 
Plusieurs femmes contribuèrent néanmoins à réinterroger les fondements d'une culture québécoise par un examen des manifestations actuelles de certaines formes d'art populaire: Lise Nantel, Louise de Grosbois et Raymonde Lamothe publièrent Les Patenteux du Québec ${ }^{8}$ suite à un travail et des activités issus d'une remise en question de l'enseignement des arts visuels, lors de l'occupation de l'École des Beaux-Arts de Montréal en 1968. C’est dans cette foulée que des liens explicites entre un art populaire traditionnel, un «art des femmes» et une forme d'art public sera élaboré par Lise Nantel et Marie Décarie dans des bannières qu'elles assemblèrent pour accompagner des manifestations politiques de toutes sortes comme signe de rassemblement des femmes. Enfin, il faut rappeler qu'aucune œuvre d'art à contenu politique ne marqua les événements d'octobre 1970 dont on connaît l’importance pour la destinée politique du Québec, sauf cette double peinture de Lise Landry présentée au sein de la Société des artistes professionnels du Québec et qui se présentait comme suit: la toile en surface intitulée «Mensonges» était recouverte d'éléments gestuels. Elle fut déchirée par l'artiste, le soir du vernissage, pour dévoiler une second toile où un patriote était peint accompagné de quelques lignes du manifeste du Front de Libération du Québec. La toile fut réquisitionnée par la police.

\section{Les femmes-artistes se regroupent}

$\underline{\text { Retour à la table des matières }}$

Dans la période effervescente du début des années 70 apparut la «question des femmes» qu'officialisera en 1975 l'Année internationale de la femme. La nécessité de repenser publiquement la position des femmes dans le tout social entraîna, sinon obligea, les institutions artistiques en place (musées, galeries) à rendre visible le travail des femmes-artistes.

8 Les Patenteux du Québec, Éd. Parti Pris, Montréal, 1974. 
La Galerie Powerhouse consacrée quasi exclusivement à la diffusion de l'art des femmes à Montréal, depuis sa fondation en 1971, répondait à un besoin urgent et à l'attente de nombreuses artistes pour qui l'accès généralisé à l'éducation et plus particulièrement à la formation en arts visuels des femmes depuis les années 60 avait fait que, dans les années 70, un nombre grandissant d'artistes était issu des écoles des beaux-arts et des universités ce qui mettait en évidence la difficulté d'accès au marché de l'art et aux institutions de diffusion pour les jeunes artistes en général et particulièrement pour les femmes.

En 1975, les institutions officielles de diffusion organiseront de grandes manifestations de groupe: «Art femme 1975» se produisit simultanément — à l'instigation de la Galerie Powerhouse et du YWCA — dans trois institutions de la métropole: le Musée d'art contemporain, le Centre Saidye Bronfman, et la Galerie Powerhouse.

En 1982, le Musée d’art contemporain présentait «Art et féminisme» exposition qui regroupait une quarantaine d'artistes dans des médiums divers et des artistes de la vidéo. Le «Réseau Art-femme» consista en des expositions et événements produits simultanément à Montréal, Sherbrooke, Québec et Chicoutimi, accueillis par les institutions officielles de diffusion en place (Musée du Québec, Galerie d'art de l'Université de Sherbrooke, Galerie de l'UQÀM); une seule se produisit dans un lieu marginal au champ artistique (Chicoutimi).

À Québec, au printemps 1984, la Galerie La Chambre Blanche dirigée majoritairement par des femmes, organisa une exposition de groupe accompagnée de manifestations diverses sous le titre «Féministe toi-même, féministe quand même»; des voies plus interrogatives que démonstratives étaient pointées, soulignant l'importance d'un questionnement renouvelé et approfondi du rapport entre art et féminisme. 


\section{Les années 70: cinq propositions}

$\underline{\text { Retour à la table des matières }}$

Contrairement à la cohérence de l'orientation stylistique des femmes-artistes des années 60, à partir des années 70 il n’y a pas d'option esthétique ni de stylistique commune pouvant catégoriser l'art des femmes (ni l'art des hommes). Aussi serait-il faux de tenter de synthétiser cette décennie par la mention d'un courant particulier ou bien de quelques noms de cheffes de file. Il n'y en eut pas et on constate en cela que les femmes-artistes s'inscrivirent dans une volonté généralisée d'éclatement des disciplines, d'introduction de l'hétérogène aux dépens de la stricte autoréférentialité dont le formalisme à l'américaine était le tenant principal. Certains noms permirent d'éclairer des positions nouvelles sur les plans esthétique et idéologique en arts: Betty Goodwin, Irene Whittome, Francine Larivée, Louisette Gauthier-Mitchell, Lise Landry. On ne peut dire que ces artistes aient été représentatives des artistes femmes des années 70, mais elles sont cependant indicatrices de positions novatrices. Ces artistes se démarquèrent des courants artistiques dominants soit par leur opposition aux rapports hommes/femmes au sein du couple traditionnel et la volonté de communication par l'art (Francine Larivée), soit par l'introduction d'éléments subjectifs et autobiographiques (Betty Goodwin, Irene Whittome), soit par le recours à une culture de femmes (Lise Landry, Louisette Gauthier-Mitchell) dans leur art.

Betty Goodwin commença un travail vraiment créateur à partir de 1969 à l'âge de 46 ans. Sa série des «Vestes» fut exposée à Montréal en 1971. Manifestement issues de sa vie personnelle, ces sortes d'icônes marquaient une orientation esthétique où l'art était fondé sur la trame intime de la vie de l'artiste. 
Un art biographique qui n’allait pourtant pas se limiter à un thème: la série des bâches fut exposée au Musée d'art contemporain de Montréal en 1976 et une autre série, celle des «Baigneurs» commencée au début des années 80 , présente une figure archétypale surgie d'expériences personnelles ${ }^{9}$. Betty Goodwin a reçu le prix P. Émile Borduas en 1986 après la seule femme artiste (Marcelle Ferron en 1983) qui ait eu cette reconnaissance avant elle.

Irene Whittome, qui s'était d'abord manifestée dans le domaine de la gravure, a situé son travail dans un espace tridimensionnel avec «Le Musée blanc» en 1975, intégrant des éléments de nature référentielle (emmaillotement, recouvrement, répétition) et archétypale. Artiste invitée à l'exposition «Québec 75» du Musée d'art contemporain de Montréal, elle s'est située d'emblée dans une perspective de rupture mais aussi d'affirmation de voies nouvelles, où les acquis de la modernité se sont pliés aux nécessités de l'intégration de la mémoire personnelle au sein du travail plastique; en 1980, des installations telles «Vancouver» et «La salle de classe», présentées lors de sa rétrospective au Musée des beaux-arts de Montréal, ont confirmé une telle orientation.

C’est dans un ordre esthétique et un contexte idéologique très différents que s'est situé le travail de Francine Larivée et celui de Louisette Gauthier-Mitchell. Aucun lien ne les relie, hormis une attention pour la figuration du couple homme/femme dans une perspective de critique et de déconstruction idéologiques.

Les gravures et dessins de Louisette Gauthier-Mitchell auxquels s'adjoignirent e 1980 des constructions tridimensionnelles en bois

9 Des dessins de cette série furent présentés à l'exposition «O Kanada» à Berlin en 1982, d'autres firent l'objet d'une installation au sein de l'exposition collective "Aurora Borealis» à Montréal en 1985. Une rétrospective de ses œuvres depuis quinze ans aura lieu au Musée des beaux-arts de Montréal en 1987. 
peints de couleurs vives 10 — sorte de tréteaux où évoluaient des figures de femmes, de couples, d'habitants d'un monde onirique et personnel - pointaient vers une mémoire collective et individuelle débordant les références particulières pour évoquer un univers épique.

C'est dans le contexte de la montée du mouvement des femmes au début des années 70 et de la nécessité de l'engagement social de l'artiste que fut réalisée et présentée la «Chambre nuptiale» sous la direction de Francine Larivée. Cette énorme tente (hauteur: 36,6 m; diamètre: 73,2 m) fut installée dans des centres d'achat de la région montréalaise (1976) et une dernière fois, en 1982, dans le cadre d' «Art et féminisme» au Musée d'art contemporain. De par sa situation excentrique, cette œuvre fut épargnée du démantèlement de Corridart ordonnée par le maire Drapeau lors des Jeux olympiques (1976). Cette œuvre se voulait un lieu et un moyen de conscientisation de l'art par la déconstruction, sur un mode à la fois épique et didactique, d'un des fondements de la société industrielle actuelle: le couple. Cet environnement soulevait la question de l'art comme moyen de communication et d'engagement social et prenait place au sein d'un questionnement plus généralisé sur l'efficacité des institutions de diffusion artistique à rejoindre un public élargi.

Enfin, le travail de Lise Landry a réhabilité des énergies et des moyens qui ont été violentés à travers des siècles d'exploitation du travail féminin. Elle a délivré des façons de faire et des gestes propres aux travaux manuels des femmes du champ de la domination économique et sociale où ils avaient été traditionnellement maintenus et, dans ses papiers tissés, cousus, épinglés, elle a exposé des valeurs positives et régénératrices habituellement exclues du domaine de l'art tout en s'inscrivant dans des problématiques actuelles telles celles du rapport support/surface, du décoratif en art, de l'éclatement des disciplines.

10 Exposition «EEuvres ouvertes 1977/1980» au Musée d’art contemporain de Montréal, 1980. 


\section{Constance de la démarche figurative chez les femmes-artistes depuis vingt-cinq ans}

Retour à la table des matières

La figuration narrative avait été adoptée par un certain nombre de jeunes artistes au début des années 70 en opposition à l'art abstrait géométrique dominant. Cette figuration avait allié des aspects d'une figuration onirique à des éléments tirés d'un univers urbain assez proche de l'esprit du Pop Art dont Louisette Gauthier-Mitchell, par exemple, s’était rapprochée.

Nous trouvons, au début des années 70, un important regain de la figuration chez un nombre important d'artistes québécois et particulièrement chez les femmes-peintres. Kittie Bruneau a joué un rôle marquant dans cette option figurative: dès les années 50, l'introduction de l'irrationnel, des figures mythiques et populaires dans des espaces picturaux bouleversés et fragmentés, avait contribué à faire de la figuration une méthode et un programme formel qui permettait d'explorer les limites du conscient et de l'inconscient tout en s'attachant à une représentation de réalités et de figures reliées autant à l'histoire personnelle de l'artiste qu'à une certaine mythologie collective.

À la fin des années 60, l'esthétique Ti-Pop avait ouvert des perspectives à la fois critiques et humoristiques sur l'environnement urbain et plus particulièrement sur les attitudes des gens en milieu urbain. Des artistes telles Michèle Bastien, Madeleine Morin, Carmen Coulombe s'y inscrivent et Josette Tsrépanier se situe dans leur continuité par la mise en scène de mythologies personnelles. Actuellement, une pratique figurative chez de plus jeunes artistes n'est pas le seul 
fait d'une participation à un courant précis, identifié au terme par trop inexact de «néo-expressionnisme».

L’option figurative a constitué à la fois une esthétique et une méthode privilégiées pour de nombreuses artistes depuis les années 60 car elle rend possible la mise à jour de l'inconscient, d'une certaine mythologie humaine et animale, des figures archétypales reliées aux cycles des retours par exemple chez Nell Tenhaff, Nicole Jolicoeur, Landon Mackenzie ou bien, comme chez Marion Wagschal, elle permet le dévoilement des codes oppressifs de la figure féminine. La peinture de Suzelle Levasseur est un exemple de l'importance accrue, depuis le début des années 80, de la gestualité expressive indissociablement liée à la représentation de la figure humaine ou animale.

Dans un tel survol, malgré l'impossibilité de les présenter adéquatement ici, il est indispensable de citer les artistes plus jeunes telles Renée Van Halm, Sylvie Bouchard, Françoise Boulet, Monique Régimblad-Zeiber, Céline Baril, Carol Wainio, chez qui la représentation figurative est investie des valeurs expressives de la gestualité. Chez Sylvie Guimont, le débordement gestuel s'est transformé en débordement de la toile dans l'espace tridimensionnel.

\section{Le geste dans l'abstraction}

\section{$\underline{\text { Retour à la table des matières }}$}

Certaines artistes ont reposé plus systématiquement la question de l'expressivité du geste en évacuant toute figuration. Si l'iconique cède le pas au pictural il ne disparaît pas nécessairement chez certaines artistes telles Hélène Roy, Francine Simonin, Louise Robert, Françoise Tounissoux. 
Hélène Roy a intégré le graphisme et la gestualité comme trame à laquelle s'intégrèrent mais sans s'y confondre, des figures de personnages et d'objets liés à sa mythologie personnelle. Contrairement, Francine Simonin - à la suite d'un long travail sur le rapport entre le geste et la forme figurative - a finalement opté, à la fin des années 70, pour l'abandon de la figure au profit du geste qui a fondé dès lors l'organisation picturale de ses toiles. Chez Louise Robert le rapport entre le geste et l'écriture, qu'on peut considérer ici comme figure, concrétisé celui du geste et du support en réduisant la couleur aux grandes oppositions de clair (geste/écriture) et de sombre (surface/support), jusqu'à récemment. Chez Françoise Tounissoux, la démarche s'est axée sur la quête du geste dans ce qu'il a d'originel, dan sa relation à la matière - ici le support est à la fois matière, surface et forme, et est considéré par l'artiste comme corps pictural au même titre que tout autre corps matériel.

\section{Sculpture, environnement, installation}

$\underline{\text { Retour à la table des matières }}$

On le sait, le domaine de la sculpture s'est difficilement ouvert aux femmes. Si le travail de certaines artistes a été répertorié il n’en reste pas moins que les femmes, travaillant avec les médiums traditionnels de cette discipline (pierre, bois, métal) ont été relativement rares.

On retrouve en 1961 comme membre fondatrices de l'Association des sculpteurs du Québec deux femmes, Ethel Rosenfeld et Yvette Bisson ${ }^{11}$ ce qui ne signifie pas néanmoins que les femmes aient été reconnues en nombre dans cette discipline. Les sculpteures furent peu nombreuses dans les expositions prestigieuses: ainsi la première Bien-

11 Cette dernière fut secrétaire de l'ASQ pendant trois ans de 1962 à 1964, de 1968 à 1969. 
nale de la sculpture canadienne, qui eut lieu à Ottawa en 1962, n'accueillit aucune sculpteure québécoise et la revue Canadian Art, qui consacrait à cette occasion un numéro spécial à la sculpture canadienne ( ${ }^{\circ}$ 80), ne mentionna qu'une seule femme: Anne Kahane. L'exposition «Panorama de la sculpture au Québec 1945-1970», qui eut lieu respectivement au Musée d'art contemporain de Montréal et au Musée Rodin (Paris) en 1970 et 1971, comprenait seulement 7 femmes sur 74 artistes ${ }^{12}$.

Ce fut plutôt par le biais de l'installation que se manifestèrent un nombre grandissant de femmes. En effet, l'installation a permis la polyvalence des matériaux et l'intégration des disciplines autrement séparées (peinture, gravure, photo, dessin, sculpture); elle a rendu désuète l'opposition historique entre abstraction et figuration et surtout a permis les références à la mémoire et à l'espace personnels.

Jusqu'au début des années 80, la méthode de Pierrette Mondou a été remarquable de simplicité et d'efficacité: elle enroulait, suspendait, disposait des tuyaux en plastique souple (de sécheuse à linge) emmaillotés de tissus ou fils dans les endroits les plus inattendus et prenait possession de l'espace et des éléments qui le structurent.

Dans une exposition solo au Musée d'art contemporain (1980), Jocelyne Alloucherie montrait une volonté de délaisser les rapports traditionnels entre peinture et sculpture, problématique qui se résoudra dans l'installation présentée dans l'exposition «Aurora Borealis» (Place de la Cité, Montréal, été 1985). Bien qu’il n’y ait pas de lien formel entre cette artiste et Hélène Gagné, ni avec Françoise Sullivan, une certaine filiation se percevait dans la recherche des mythes premiers, les références à l'irrationnel, et à l'inconscient vu comme source véritable de rationalité.

12 Les sculpteures étaient: Yvette Bisson, Lise Gervais, Suzanne Guité, Claire Hogenkamp, Anne Kahane, Ethel Rosenfeld, Françoise Sullivan. 
Dans un tout autre registre et dans des matières souples et anciennes (le feutre, les fibres de papier), Michèle Héon plonge dans la mémoire pour y saisir des formes significatives tels ses manteaux dressés qui réfèrent à une présence immémoriale, celle des femmes. Travaillant un matériau souple mais dur (le béton moulé), les sculptures de Tatiana Demidoff évoquent un espace et un temps mythiques, incluent une dimension anthropologique circonscrite dans les gestes et les parcours rituels que d'autres artistes ont également inscrits dans leur méthode créatrice.

Brigitte Radecki pointe une dimension de l'espace archaïque dans l'édification de ses enclos, rappelant les lieux sacrés tracés au sol par les augures. Dans une telle perspective, Françoise Sullivan apparaît comme celle qui a inauguré l'exploration de l'inconscient par la référence à l'archaïque, aux mythes, aux cycles naturels de croissance et de déclin, alliant ses propres gestes et sa mémoire intime aux rites de cultures anciennes et disparues et cela tant dans ses sculptures que dans ses installations et ses performances.

Le domaine de la mémoire intime et singulière a été exploré par deux artistes qui se produisent et travaillent ensemble: Manon Thibault, sculpteure, Ginette Prince, danseuse et photographe, réalisent des environnements et performances où l'intégration de disciplines diverses permet la mise en scène d'univers intimes et ésotériques.

La rencontre et la pénétration réciproque du monde de l'inconscient et du monde de la vie quotidienne a été la dimension majeure des environnements de Blanche Celanuy et de Barbara Steinman: chez cette dernière, l'image vidéo tient lieu de présence et d'absence tout à la fois dans des installations présentant des lieux désertés et anonymes. Monique Mongeau parle également de silence dans ses mises en scène où l'espace pictural et l'espace réel se confondent, jouant sur la fiction et la mémoire d'un lieu autre 13 .

13 Exposition collective «Actuelles», Place Ville-Marie, Montréal, 1983. 
À partir de 1983, c'est dans la dialectique nature/culture soustendus par une pensée écologique, que Francine Larivée a renoué avec les lieux publics, y amenant des environnements modulaires où des mousses vivantes, fixées sur une base en polystyrène, forment des territoires fictifs et utopiques d'où toute figure humaine est absente.

Comme plusieurs artistes qui ont ouvert leurs ateliers au public depuis le début des années 80 à Montréal afin d’y présenter leur travail dans et sur le lieu réel, Diane Gougeon et Eva Brandl ont plusieurs fois présenté leurs installations, qu'elles soient permanentes ou éphémères, dans les lieux mêmes de leur travail. La modification physique d'un logement de la rue Mentana (Montréal, 1978-1980) par Betty Goodwin fut un précédent qui ouvrit la voie à plusieurs réalisations: ainsi, deux artistes qui travaillent ensemble depuis quelques années, Martha Fleming et Lyne Lapointe, ont orienté leurs interventions sur des édifices abandonnés ou du moins inhabités ${ }^{14}$, matérialisant ainsi la critique institutionnelle qui fonde en partie leur travail artistique. Cette distance prise par rapport aux lieux institutionnels de diffusion questionne directement la légitimité des savoirs. Dans «Le Musée des Sciences», en collaboration avec Madeleine Jean, ces artistes posaient le rapport explicite entre l'aliénation du corps féminin et le pouvoir médical et scientifique.

Ces interventions constituent un pôle limite où le projet créateur est fondé en partie sur la modification des données architectoniques des lieux institutionnels. Elle mettent au jour des rapports autrement cachés entre la structure des édifices et l'organisation de l'imaginaire social et individuel.

14 Caserne des pompiers n 14, rue Saint-Dominique à Montréal en janvier 1983. «Le Musée des Sciences», au Bureau de poste, rue Notre-Dame Ouest, février 1984. 
Pour leur part, Geneviève Cadieux et Sandra Meigs, dans des installations spectaculaires mettent en scène des figures qui dévoilent des aspects de leur inconscient et de leur imaginaire 15 .

\section{Photo, vidéo, performance}

$\underline{\text { Retour à la table des matières }}$

Nous ne pouvons aborder ici la photographie sous son angle descriptif et communicationnel. C'est sous celui d'un travail sur le médium que nous pouvons ici la signaler. Cette approche a été pratiquée d'une façon remarquable par Sorel Cohen, Raymonde April, Freda Guttman chez qui le temps, si profondément inscrit dans la matérialité du médium photographique, et l'espace sont intrinsèquement liés.

Dans le domaine de la vidéo, Marshalore, dès le milieu des années 70 à Montréal, a eu un rôle marquant, tant du point de vue de la production comme telle que par sa contribution à l'organisation d'un réseau. Ann Ramsden, Joyan Saunders, Barbara Steinman, Corinne Corry, Colette Tougas, Michèle Waquant, ont produit des bandes qui témoignent de ce travail formel sur le médium, tout en maintenant une signification ayant trait au rapport intime de l'image de soi aux codes et stéréotypes sociaux qui ont été dénoncés et déconstruits.

Dans le domaine de la performance, au Québec, les femmes ont été peu nombreuses à se manifester: Marie Chouinard, Francine Chaîné, Sylvie Laliberté, Sylvie Tourangeau, entre autres issues de la danse, des arts visuels, du théâtre, représentent les approches qui ont marqué ce domaine d'une façon substantielle.

15 Exposition de groupe «Avant-scène de l'imaginaire», Musée des beaux-arts de Montréal, hiver 1985. Cette exposition regroupait Françoise Boulet, Geneviève Cadieux, Sandra Meigs. 
L'intérêt remarquable qu'a soulevé l'exposition «Montréal ToutTerrain» (Montréal, été 1984) a fait de cet événement un «précédent». Son comité d'organisation était composé de huit femmes et a réuni une soixantaine d'artistes dont la moitié était des femmes ${ }^{16}$. Si on s'en remettait à l'importance du nombre de femmes et à celle des œuvres présentées à l'exposition «Montréal Tout-Terrain ${ }^{17}$ », on pourrait croire que la présence des femmes est dorénavant assurée et reconnue au Québec. Il ne faut pas oublier néanmoins qu'en 1978, une enquête ${ }^{18}$ soulignait le fait que les femmes n'occupent pas plus du quart du nombre des artistes qui sont exposés dans les musée, les galeries, qui font partie des collections publiques et privées au Canada. Et encore, ce quart est-il la proportion maximale de femmes-artistes dans ces différents secteurs.

En réalité, il est nécessaire de réévaluer les mouvements et courants artistiques comme tels en regard même de la place que les femmes-artistes y ont ou n'y ont pas occupée, en tenant compte du contexte social et historique dont elles font partie. Qui plus est, audelà de questions stylistiques, ce sont les idéologies sous-tendant de façon voilée tel ou tel courant qui sont à mettre à jour, elles débordent la seule structure formelle de l'œuvre en tant qu'objet.

16 Des installations de Danielle Sauvé, Louise Viger, Renée Chouinard, Deborah Margo \& Cynthia Van Frank, Ilana Isehayek, des montages de Monique Régimbald-Zeiber, Céline Baril, Mary-Ann Cuff, Sylvie Guimont entre autres, témoignaient d'une maîtrise remarquable de leurs moyens d'intervention et de leurs médiums, mettant à jour un imaginaire où les domaines de l'inconscient, de la mémoire intime, se croisaient.

17 «Montréal Tout-Terrain», au 305 Mont-Royal Est, Montréal, été 1984. Le comité d'organisation de l'exposition était composé de Céline Baril, Sylvie Bouchard, Janine Fisher, Christiane Gauthier, Diane Gougeon, Lesley Johnstone, Martine Meilleur, Claire Paquet.

18 Avis Lang Rosenberg, «Women artists and the Canadian Art World: a Survey», Criteria, vol. 4, n² , automne 1978, p. 13-18. 
Par le biais d'expositions qui ont été respectivement présentées à Montréal («Via New York», au Musée d’art contemporain en 1984) et à Toronto («The European Iceberg», Art Gallery of Ontario, en 1985), on peut mesurer la chute spectaculaire de la présence des femmes dans les grandes expositions internationales: cela fut signalé dès le début des années 80 et des manifestations en ont dénoncé la tendance réactionnaire. En général, en Europe et en Amérique du Nord, les femmes qui avaient acquis une renommée certaine sont de moins en moins visibles malgré le renouvellement et l'importance de leur travail artistique. Pour le Québec, cela est un avertissement où, malgré des acquis certains, on ne peut parler d'irréversibilité: il faut se rappeler qu'une exposition telle «Repères, art actuel du Québec» organisée par le Musée d'art contemporain de Montréal en 1982 et présentée à travers le Canada jusqu'en 1984, n'incluait qu'une femme sur dix artistes.

Peu ou pas d'études ont été faites sur l'histoire de la présence des femmes-artistes dans le champ artistique au Québec 19 et leur apport spécifique. Les bilans sont trompeurs: celui-ci l'est ni plus ou moins qu'un autre car tout bilan chronologique détermine une linéarité qui limite, emprisonne sous un angle de vue nécessairement restreint la complexité qui sous-tend les démarches, les concepts, les engagements. Aussi faut-il ouvrir dans cette surface lisse des faits historiques une brèche: ayant ici inscrit certains noms, divers faits et événements qui autrement risquent d'être oubliés faute d'être renommés soit parce que n'allant dans le sens de la «marche officielle» de l'histoire de l'art, soit parce que non considérés comme déterminants, il faut marquer l'importance de certaines œuvres et certaines approches de femmes-artistes dont certaines ont à voir avec la psychanalyse (dans les œuvres de Nicole Jolicoeur et d'Isabelle Bernier par exemple), la sémiologie (dans celles de Jocelyne Alloucherie), l'anthropologie (chez

19 Un survol rapide a été tracé en 1975 par Rose-Marie Arbour et Suzanne Lemerise dan «Le rôle des Québécoises dans les arts plastiques depuis trente ans», Vie des Arts, vol. XX, n 78, printemps 1975, p. 16-24. 
Irene Whittome); d'autres se tournent vers des instruments tel l'ordinateur (Nell Tenhaff), vers des exigences d'une pensée écologique (Francine Larivée) et systématiquement critiquent l'histoire et la constitution des savoirs de domination (Lyne Lapointe, Martha Fleming). Les cloisonnements ne tiennent plus, ni entre les disciplines artistiques, ni entre ce qui est «art» et ce qui ne l'est plus. Si une mémoire est remise à flot elle ne constitue pas pour autant le but de la pratique artistique: elle est posée au sein des conventions formelles et esthétiques actuelles et de ce fait, les modifie, les ouvre à d'autres sens.

Plusieurs artistes ont puisé dans les modes d'approche et d'existence d'une culture propre aux femmes de nouveaux indices, elles ont de ce fait accédé à d'autres pistes, d'autres points de vue sans lesquels leur pratique actuelle n'aurait pu se définir ni s'orienter. Cette mémoire redécouverte n'est significative cependant qu'en fonction même de ses possibilités à ouvrir de nouveaux domaines, à mettre à jour des modes de perception, une conscience d'où les hiérarchies et les décloisonnements ont sinon entièrement disparu, du moins ont été profondément perturbés et mis en cause.

La position des femmes-artistes dans le système de l'art et dans le tout social leur permet d'orienter leur projet créateur en fonction de valeurs autres dont on peut voir l'affirmation chez plusieurs artistes et qui, si assumée collectivement mais non uniformément ou dogmatiquement, permet et permettra de modifier la conscience artistique en proportion même de la modification d'une conscience individuelle.

Fin du texte 